\title{
Immunohistochemical correlates of TP53 somatic mutations in cancer
}

\author{
Balázs Murnyák ${ }^{1}$ and Tibor Hortobágyi ${ }^{1}$ \\ ${ }^{1}$ Division of Neuropathology, Institute of Pathology, Faculty of Medicine, University of Debrecen, Debrecen, Hungary \\ Correspondence to: Tibor Hortobágyi, email: hortobagyi@med.unideb.hu \\ Keywords: IARC TP53 Database, immunohistochemistry, TP53 mutations, cancer, p53 \\ Received: June 17,2016 Accepted: September 01, 2016 Published: September 08, 2016
}

\section{ABSTRACT}

Despite controversy on the correlation between p53 accumulation and TP53 mutational status, immunohistochemical (IHC) detection of overexpressed protein has long been used as a surrogate method for mutation analysis. The aim of our study was to characterise the IHC expression features of TP53 somatic mutations and define their occurrence in human cancers. A large-scale database analysis was conducted in the IARC TP53 Database (R17); 7878 mutations with IHC features were retrieved representing 60 distinct tumour sites. The majority of the alterations were immunopositive $(p<0.001)$. Sex was known for 4897 mutations showing a female dominance $(57.2 \%)$ and females carrying negative mutations were significantly younger. TP53 mutations were divided into three IHC groups according to mutation frequency and IHC positivity. Each group had female dominance. Among the IHC groups, significant correlations were observed with age at diagnosis in breast, bladder, liver, haematopoietic system and head \& neck cancers. An increased likelihood of false negative IHC associated with rare nonsense mutations was observed in certain tumour sites. Our study demonstrates that p53 immunopositivity largely correlates with TP53 mutational status but expression is absent in certain mutation types. Besides, describing the complex IHC expression of TP53 somatic mutations, our results reveal some caveats for the diagnostic practice.

\section{INTRODUCTION}

The TP53 gene $(17 \mathrm{p} 13)$ encodes a nuclear transcription factor expressed in response to various stress signals such as DNA damage, heat shock, hypoxia, and oncogene overexpression. Upon activation, p53 maintains the integrity and stability of the genome by triggering cell-cycle arrest, DNA repair, and apoptosis [1]. The 393-amino-acid tumour-suppressor protein consists of an N-terminal transactivation domain (residues 1-42), a proline-rich domain (residues 40-92), a sequence specific core DNA binding domain (residues 103-306), a tetramerization domain (residues 307-355) and a C-terminal regulatory domain (residues 356-393) [2].

While other tumour suppressor genes are inactivated by mutations leading to absence of protein, TP53 alterations are usually missense producing stable fulllength protein [3]. Somatic TP53 mutations primarily occur at the 175, 245, 248, 249, 273 and 282 'hotspot' codons in the conserved DNA binding domain (DBD), but they can also happen in any other sites within the gene [4]. Extensive efforts have been made to study TP53 mutation effect on prognosis, therapeutic response and its role in cancer diagnosis. It was previously reported, that p53 overexpression is a prognostic indicator in colorectal, lung, prostate, and breast carcinomas [5-7].

Fast and reliable detection is crucial for the accurate diagnostic decisions and targeted therapies [8]. There is an agreement that overexpressed $\mathrm{p} 53$ protein indicates the presence of TP53 alterations [9]. Wild-type p53 is an unstable protein with a short half-life for its detection by immunohistochemistry (IHC), but mutant p53 can accumulate within tumour cells creating a stable target for IHC [10]. Although nucleotide sequencing is the gold standard to identify TP53 mutations, due to its beneficial features IHC has long been used as a surrogate method for mutations analysis in histopathological diagnostic practice [11]. 
Recent tumour profiling and data sequencing databases enable to more complex research, including molecular epidemiology, clinical surveys, and structural analyses $[12,13]$. The International Agency for Research on Cancer (IARC) TP53 Mutation Database (http://p53. iarc.fr/) contains data on the prevalence and patterns of more than 28000 somatic mutations in human cancers, annotations of tumour phenotype, patient characteristics, the structural and functional impact, and immunostaining of the mutations. The database includes all published TP53 mutations, all confirmed by sequencing, published in the peer-reviewed journals or compiled in mutation data repositories [1,14].

The aims of our study were to characterise the IHC expression features of TP53 somatic mutations, and define their occurrences in human cancers. For this purpose, a large-scale analysis has been conducted involving the available p53 IHC and clinicopathological data in the IARC TP53 Database. Besides, describing the IHC expression characteristics of TP53 mutations, our results revealed some caveats of using p53 IHC as a surrogate for mutation analysis.

\section{RESULTS}

\section{IHC expression characteristics of somatic mutations}

Altogether, p53 IHC data was available in 7878 mutations, representing $26.5 \%$ of the 29711 TP53 somatic mutations in the IARC Database. The IHC staining was positive in 6026 mutations (76.5\%), whereas 1852 mutations $(23.5 \%)$ were negative by IHC $(p<0.001)$. A key purpose of this study was to provide a complex characterisation of IHC patterns of TP53 mutations. Regarding mutation types, single nucleotide alterations were predominantly IHC positive at a range between $74.9 \%$ and $84.6 \%$; tandem (93.8\%) and complex mutations were also positive, whereas deletions and insertions were mostly negative (57\% and $59.7 \%$, respectively). As expected, $88 \%$ of missense mutations were p53 IHC positive, whereas $71.2 \%$ of nonsense mutations were negative for IHC $(p<0.001)$.

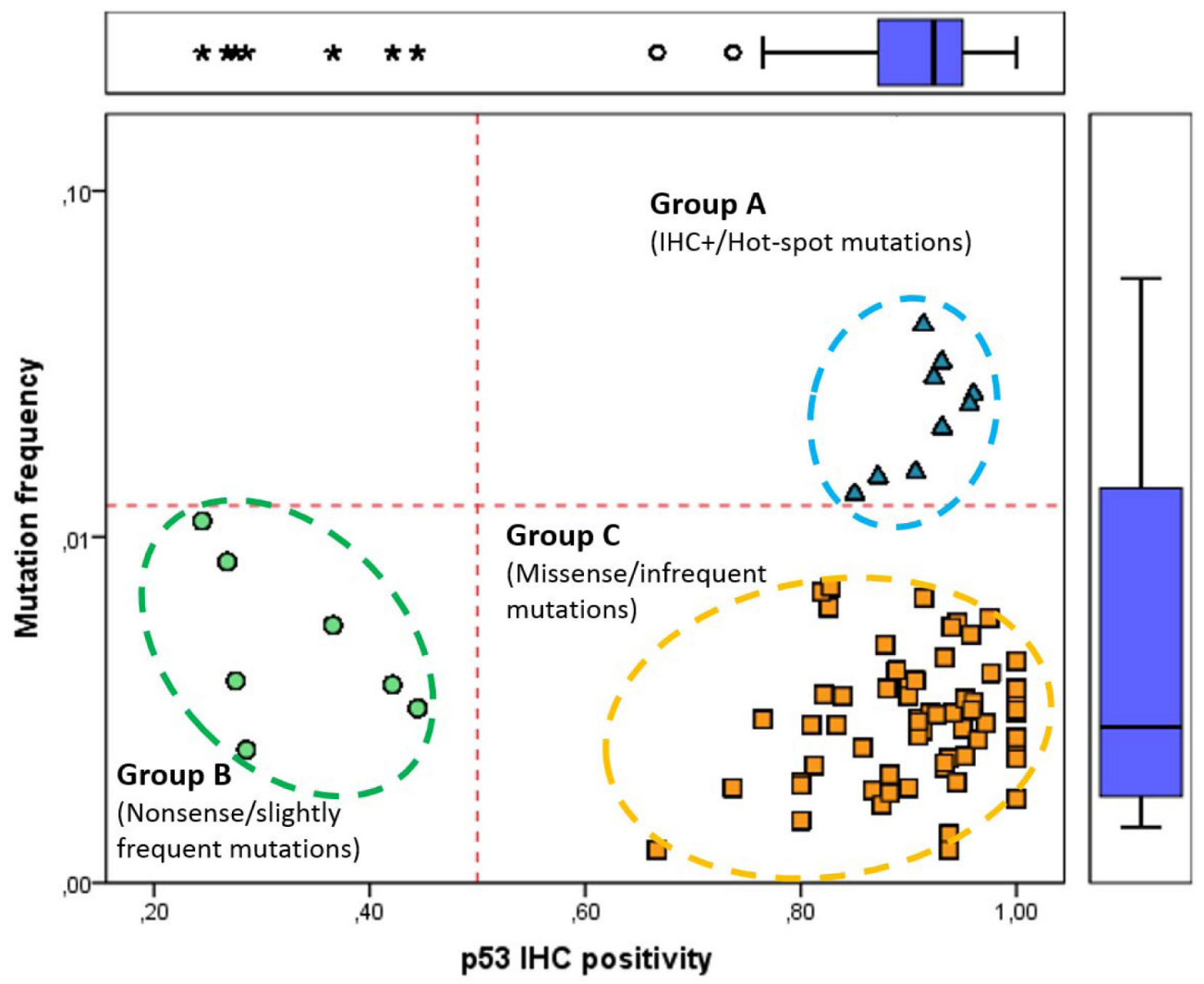

Figure 1: Classification of TP53 somatic mutations based on frequency and IHC positivity in IARC TP53 Database. Somatic TP53 alterations were categorized into three groups: 'hot spot' mutations (marked with blue triangle) are frequent in Group A (mutation frequency $\geq 0.013$ and IHC positivity $\geq 0.85$ ); common nonsense mutations (blue circles) are in Group B (mutation frequency $\leq 0.011$ and IHC positivity $\leq 0.44$ ); less frequent and mostly positive missense mutations (yellow square) fall into Group C (mutation frequency $\leq 0.072$ and IHC positivity $\geq 0.74$ ). Each data point represents distinct TP53 mutations and only individual mutations with 15 or more IHC results were considered. 
Table 1: Immunohistochemical expression characteristics of TP53 mutations.

\begin{tabular}{|c|c|c|c|c|c|c|}
\hline \multirow{3}{*}{\begin{tabular}{|l}
$\begin{array}{l}\text { Mutation } \\
\text { features }\end{array}$ \\
Exons/introns \\
\end{tabular}} & \multicolumn{5}{|c|}{ p53 immunohistochemistry } & \multirow{3}{*}{$p$-value } \\
\hline & \multirow[t]{2}{*}{ Total $(n=7878)$} & \multicolumn{2}{|c|}{ Negative $(\%)(n=1852)$} & \multicolumn{2}{|c|}{ Positive $(\%)(n=6026)$} & \\
\hline & & & & & & \\
\hline exons & 7690 & 1745 & $(22.69)$ & 5945 & $(77.31)$ & \multirow{3}{*}{0.001} \\
\hline introns & 186 & 105 & $(56.45)$ & 2086 & $(43.55)$ & \\
\hline NA & 2 & 2 & $(100.00)$ & 0 & $(0.00)$ & \\
\hline \multicolumn{7}{|l|}{ CpG site } \\
\hline no & 5373 & 1433 & $(26.67)$ & 3940 & $(73.33)$ & $<0.0001$ \\
\hline yes & 2505 & 419 & $(16.73)$ & 2086 & $(83.27)$ & $<0.0001$ \\
\hline \multicolumn{7}{|l|}{ Splice site } \\
\hline alternative & 71 & 12 & $(16.90)$ & 59 & $(83.10)$ & 0.1872 \\
\hline consensus & 112 & 72 & (64.29) & 40 & $(35.71)$ & $<0.0001$ \\
\hline criptic & 62 & 16 & $(25.81)$ & 46 & (74.19) & 0.6684 \\
\hline no & 7633 & 1752 & $(22.95)$ & 5881 & $(77.05)$ & $<0.0001$ \\
\hline \multicolumn{7}{|l|}{ Type } \\
\hline Single nucleotide mutations ${ }^{\mathrm{b}}$ & 6860 & 1324 & (19.30) & 5536 & $(80.70)$ & $<0.0001$ \\
\hline tandem & 97 & 6 & $(6.19)$ & 91 & $(93.81)$ & 5.16E-05 \\
\hline complex & 48 & 18 & $(37.50)$ & 30 & $(72.50)$ & 0.0219 \\
\hline deletion & 667 & 380 & (56.97) & 287 & (43.03) & $<0.0001$ \\
\hline insertion & 196 & 117 & $(59.69)$ & 79 & $(40.31)$ & $<0.0001$ \\
\hline NA & 10 & 7 & \begin{tabular}{|l|}
$(70.00)$ \\
\end{tabular} & 3 & $(30.00)$ & 0.000522 \\
\hline \multicolumn{7}{|l|}{ Effects } \\
\hline frameshift & 677 & 451 & (66.62) & 226 & $(33.38)$ & $<0.0001$ \\
\hline intronic & 53 & 25 & $(47.17)$ & 28 & $(52.83)$ & 4.58E-05 \\
\hline large deletion & 2 & 2 & $(100.00)$ & 0 & $(0.00)$ & 0.0107 \\
\hline missense & 5889 & 707 & (12.01) & 5182 & (87.99) & $<0.0001$ \\
\hline nonsense & 552 & 393 & $(71.20)$ & 159 & $(28.80)$ & $<0.0001$ \\
\hline silent & 367 & 136 & $(37.06)$ & 231 & $(62.94)$ & $3.64 \mathrm{E}-10$ \\
\hline splice & 131 & 86 & $(65.65)$ & 45 & $(34.35)$ & $<0.0001$ \\
\hline other & 177 & 44 & $(24.86)$ & 133 & $(75.14)$ & 0.6683 \\
\hline NA & 30 & 8 & $(26.67)$ & 22 & (73.33) & 0.6828 \\
\hline \multicolumn{7}{|l|}{ Structural motif } \\
\hline C-terminal & 234 & 104 & (44.44) & 130 & $(55.56)$ & $<0.0001$ \\
\hline C- terminal/NLS & 31 & 13 & $(41.94)$ & 18 & $(58.06)$ & 0.0153 \\
\hline C- terminal/tetramerization & 101 & 39 & $(38.61)$ & 62 & $(61.39)$ & 3.15E-04 \\
\hline $\mathrm{L} 1 / \mathrm{S} / \mathrm{H} 2$ & 1693 & 234 & $(13.82)$ & 1459 & (86.18) & $<0.0001$ \\
\hline L2/L3 & 2765 & 434 & $(15.70)$ & 2331 & $(84.30)$ & $<0.0001$ \\
\hline N- terminal & 34 & 26 & $(76.47)$ & 8 & $(23.53)$ & $<0.0001$ \\
\hline $\mathrm{N}$ - terminal/Transactivation & 33 & 20 & $(60.61)$ & 13 & $(39.39)$ & $<0.0001$ \\
\hline N- terminal/Transactivation/ NES & 5 & 2 & $(40.00)$ & 3 & $(60.00)$ & 0.3844 \\
\hline NDBL/ $\beta$-sheets & 2657 & 795 & $(29.92)$ & 1862 & $(70.08)$ & $<0.0001$ \\
\hline SH3-like/Proline-rich & 101 & 60 & $(59.41)$ & 41 & $(40.59)$ & $<0.0001$ \\
\hline NA & 224 & 125 & $(55.80)$ & 99 & $(44.20)$ & $<0.0001$ \\
\hline
\end{tabular}

${ }^{\mathrm{a}}$ Comparisons between groups were performed with Pearson's Chi-square $\left(\chi^{2}\right)$, Significant $p$-values are marked in bold; ${ }^{\mathrm{b}} \mathrm{Containing}$ the following point mutations: $\mathrm{A}: \mathrm{T}>\mathrm{C}: \mathrm{G}, \mathrm{A}: \mathrm{T}>\mathrm{G}: \mathrm{C}, \mathrm{A}: \mathrm{T}>\mathrm{T}: \mathrm{A}, \mathrm{G}: \mathrm{C}>\mathrm{A}: \mathrm{T}, \mathrm{G}: \mathrm{C}>\mathrm{A}: \mathrm{T}$ at $\mathrm{CpG}, \mathrm{G}: \mathrm{C}>\mathrm{C}: \mathrm{G}$ and $\mathrm{G}: \mathrm{C}>\mathrm{T}: \mathrm{A})$ 
Almost eighty percent $(77.3 \%)$ of the mutations were positive within the coding regions (e.g. exons 2-11), whereas the majority of alterations in the non-coding sequences were negative $(56.5 \%)$ for IHC $(p=0.001)$ (Supplemental Figure S1). Mutations outside and within the $\mathrm{CpG}$ island regions were positive in $83.3 \%$ and $73.3 \%$, respectively $(p<0.001)$. TP53 mutations were positive in non-splice site sequences as well as in alternative and cryptic sites $(77 \%, 83.1 \%$ and $74.2 \%$, respectively), but $64.3 \%$ of the mutations were negative in the consensus splice sites. The IHC expression patterns were diverse among the structural motifs of mutated p53.

Mutations were unequivocally positive in $\mathrm{L} 1 / \mathrm{S} / \mathrm{H} 2$ (86.2\%), L2/L3 loops (84.3\%), and NDBL/beta-sheets motifs $(70.1 \%)$. Furthermore, alterations were positive in the C-terminal (55.6\%), C-terminal/NLS (58.1\%), and $\mathrm{N}$-terminal/Transactivation/NES $(60 \%)$ motifs. On the other hand, TP53 mutations were mostly negative in the N-terminal (76.5\%), N-terminal/Transactivation (60.6\%), and SH3-like/Proline-rich (59.4\%) motifs. The structural motifs of 224 mutations were non-available (Table 1).

\section{IHC expression patterns of individual somatic TP53 mutations}

Our other purpose was to describe the IHC expression profile of individual TP53 mutations and compare their clinico-pathological features in various tumour sites. First, we estimated the frequencies of each 1778 individual TP53 mutations in the IARC database. Second, the IHC results were retrieved from the database. IHC expression patterns were available of 1139 individual TP53 mutations. Finally, we associated the alterations with their expression patterns. IHC data and frequencies of TP53 somatic mutations are summarized in Supplemental Table S1.

The majority of the mutations in hotspot p53 codons were strongly positive: 175 (89.7\%), 245 (90.7\%), 248 (93.3\%), 249 (88.0\%), 273 (92.6\%) and 282 (90.9\%). In contrast, mutations were usually negative among the frequently altered codons like $213(57.7 \%), 196(65.2 \%)$, $306(60.9 \%), 146(63.6 \%)$ and 298 (60.7\%). More specifically, all alterations within the hotspot codons (R175H, G245S/D, R248Q/W/L, R249S, R273H/C/L, and $\mathrm{R} 282 \mathrm{~W}$ ) were positive for $\mathrm{p} 53 \mathrm{IHC}$ at a range of 87.2$100 \%$. Contrary, frequent nonsense mutations such as R213*, R196*, R306*, W146*, and E298* were not able to be detected (63.4-75.6\%) by immunohistochemistry.

Based on the mutation frequencies and IHC positivity, TP53 mutations possess more than $15 \mathrm{IHC}$ data $(n=78)$ were categorized into three distinct groups. Hotspot and IHC positive mutations $(9 / 78 ; 11.5 \%)$ were in Group A, frequent and mostly IHC negative mutations $(7 / 78 ; 9 \%)$ were in Group B, whereas infrequent and IHC positive mutations were in Group C $(62 / 78 ; 79.5 \%)$

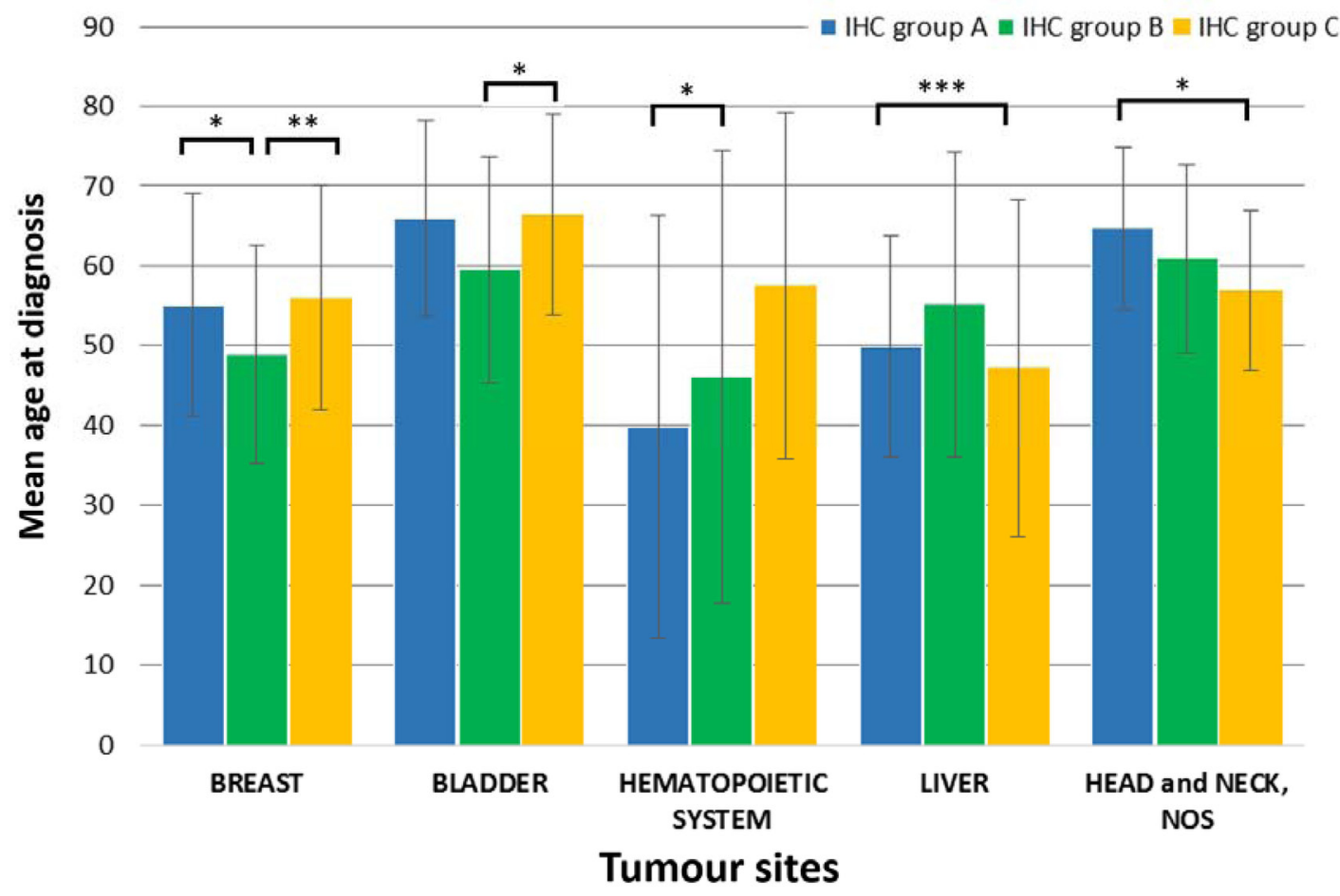

Figure 2: Significant differences of mean age of TP53 mutation carriers between IHC groups were found in five tumour sites. Bar graphs represent data in mean \pm standard deviation (SD). Level of significance: $*(p<0.05), * *(p<0.01)$, and *** $(p<0.001)$. 
(Figure 3). Principal components analysis of the groups by two components also confirmed differences between the three groups (Supplemental Figure S3). All alterations in Group A and C were missense, whereas in Group B they were nonsense $(p<0.001)$. The majority of TP53 mutations in Group A and B localized within exons 5-8 $(p<0.001)$, and they commonly occurred in the $\mathrm{CpG}$ sequences $(p<0.001)$.

\section{Clinicopathological features of p53 IHC groups}

Sex was available for 4897 TP53 mutations with known IHC data, including 2797 females (57.2\%) and 2097 males (42.9\%). The p53 IHC was predominantly positive $(75.8 \%$ vs. $76.4 \%)$ in both sex but females with
IHC negative mutations were significantly younger $(55.90$ \pm 15.391 vs. $58.57 \pm 15.062$ years, $p=0.0182)$. There was no significant difference regarding the mean age (56.44 $\pm 16.86 v s .57 .22 \pm 15.772, p=0.204)$. TP53 alterations were IHC positive in almost all human cancers in the database at a range between 50 and $100 \%$.

Sex distribution and the mean age at diagnosis were also evaluated in the three p53 IHC groups. A significant female dominance was observed in all groups $(p=0.0042)$. Significant differences were observed in five tumour sites (breast, bladder, haematopoietic system, liver, head and neck) in term of the age at diagnosis among the IHC groups (Figure 2). Breast cancer patients carrying nonsense mutations (Group B) were significantly younger $(48.83 \pm 13.69$ years) than patient in the other groups

Frequent nonsense TP53 mutations (IHC Group B)

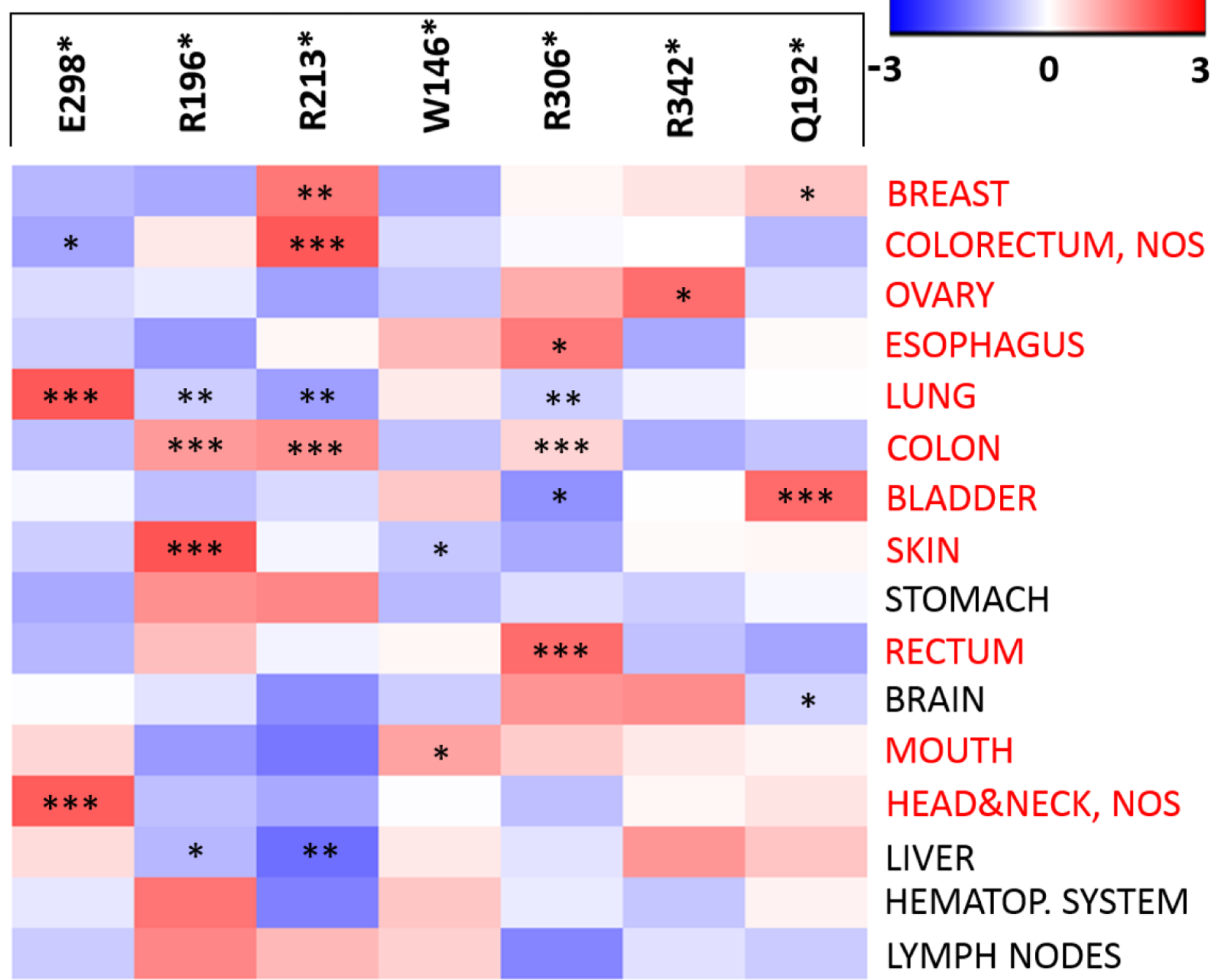

Figure 3: Frequency differences of nonsense TP53 (Group B) mutations across tumour sites. The 'heat map' shows the different frequency of the seven nonsense TP53 mutations (ranging from -3 to 3) across tumour sites (compared to their overall frequency in IARC database). A heat-map of nonsense TP53 mutations were constructed using Gene-E version 3.0.204 (http://www.broadinstitute. org/cancer/software/GENE-E/index.html). Level of significance: $*(p<0.05),{ }^{* *}(p<0.01)$, and $* * *(p<0.001)$. 
(Group A: 55.06 \pm 13.99 years \& Group C: $55.97 \pm 14.07$ years). Similar difference was observed in bladder cancer, but patients were notable younger in only Group B (59.52 \pm 14.20 years) compared to Group A $(64.44 \pm 12.57$ years). Contrary, liver carcinoma patients in Group A were significantly older then in Group C. Comparison of the Group $\mathrm{A}$ and $\mathrm{C}$ revealed that patients with frequent missense mutations were younger with haematopoietic system, but older with head and neck tumours. Mean ages in different IHC mutation groups are summarized in Supplemental Table S3.

Because the majority of nonsense TP53 mutations were undetectable by IHC and carriers usually have worse overall survival and a poor prognosis, further characterisation of nonsense mutations in Group B was also important. Significant differences were observed between the total frequencies of the mutations in the IARC Database and their frequency in several tumour sites (Figure 3). For example, R213* was more common in breast, colorectum, colon, and less frequent in liver, lung compared to its overall frequency. Frequencies of nonsense mutations at various tumour sites are summarized in Supplemental Table S4.

\section{DISCUSSION}

Despite the numerous studies, the association between TP53 mutations and p53 nuclear accumulation is still not fully understood. Although immunohistochemistry is commonly used as a surrogate for TP53 mutation analysis in the routine diagnostic work-up, its reliability is controversial due to the false-positive and negative cases. The aim of our study was to estimate the immunohistochemical expression patterns of TP53 somatic mutations at various tumour sites and correlate them with clinicopathological features. Therefore, we performed a comprehensive evaluation of the R17 dataset from the IARC TP53 Database $[1,14]$.

In normal conditions, synthesis and degradation of p53 are strictly regulated, its expression level is maintained very low [15]. Aberrant p53 has a prolonged turnover resulting in an overexpressed protein that can be detected by IHC [16]. Our study confirm that more than $75 \%$ of TP53 mutations showed IHC-detectable p53 accumulation in a unified manner. This absolute positivity was also observed in all tumour sites. This is in contrast with other tumour suppressor genes where mutations are mostly deletions or nonsense leading to a decreased or absent protein expression [3].

The majority of TP53 mutations are predominantly clustered in the DNA binding domain (residues 94-292) [1]. In our results, over $94 \%$ of the IHC positive alterations also occurred in this domain (Supplemental Figure S2). The DNA-binding domain includes three particularly important regions. The L2 loop (codons 163-195) is required for the folding and stabilization of the central part of the protein, whereas L3 loop (codons 236-251) and loop-sheet-helix (L1/S/H2) motif (codons 273-286) can contact the DNA at least two residues (241, 248 and 273,280 , respectively). TP53 mutations in these domains are associated with a more aggressive clinical phenotype, presumably they decrease biological activity of the protein $[17,18]$. As indicated in Table 1, mutations within these crucial motifs are mostly positive by IHC.

The majority of TP53 mutations occur within exons 5-8 [4]. In our study, more than $95 \%$ of the alterations localized within these exons and showed IHC positivity. Point mutations in TP53 introns can abrogate the function of any remaining normal p53 [19]. Our findings are consistent with previous reports, because $87 \%$ of the IHCdetectable mutations were single base-pair substitutions in coding regions. These are missense mutations that usually produce full-length proteins, consequently, more than $80 \%$ of the alterations were also IHC positive according to the IARC database. Besides, the majority of tandem and complex TP53 mutations are also IHC positive, however, they are relatively infrequent in human cancer [1]. Splice mutations in the TP53 are reported as infrequent events. Accordingly, only the $3 \%$ of the mutations occurred within the conserved dinucleotides involved in splice sites and their distribution and IHC characteristics were diverse. The altered transcript is sufficiently stable and not degraded [20]. Splicing alterations in the alternative and cryptic splice acceptor sites showed IHC positivity; in contrast, they were negative in consensus splice sites. There was no remarkable difference in IHC expressions with respect to the position of the mutation within or outside $\mathrm{CpG}$ sequences, both of them were mostly positive (in $83.3 \%$ and $73.3 \%$, respectively).

We also analysed the association between individual TP53 mutations and their IHC characteristics. For this reason, somatic TP53 mutations in the IARC database were classified into three groups based on their frequency and IHC positivity. The "hotspot" mutations (Group A) and frequent nonsense mutations (Group B) formed distinct groups, whereas the infrequent strongly positive missense mutations were in Group C (Figure 1). The key starting point of our study was that nonsense TP53 mutations (Group B) result in lack of immunolabelling due to the absence of gene product [21]. Truncating mutations of genes like $N F 2$ or $H N F 1 A$ are associated with an early age of onset $[22,23]$. However, TP53 mutations were predominantly IHC positive in both sexes. Females with IHC negative mutations (manly caused by truncating mutations) were younger. According to our classification of TP53 mutations, the age at diagnosis of Group B was lower in breast and bladder cancers, and interestingly, it was higher in tumours of the haematopoietic system compared to Group A and C. The frequency of R213* and Q192* was also increased in breast, as well as R306* and Q192* mutations frequency in bladder cancer. In breast cancer, R213* TP53 mutations are more frequent in the 
basal-like subtype [17]. In colon cancer, R196*, R213* and R306* mutations were also common. Furthermore, R213* mutation was common in colorectum, and R306* in rectum carcinomas. Vakiani et al. reported, that R306*mutation was present in $15.27 \%$ and $39.5 \%$ of alleles in the invasive component of the primary tumour and in the metastasis, respectively [18]. The W146* mutation was slightly more frequent in skin and mouth cancers in our analysis.

Although nonsense (Group B) mutations are infrequent, they indicate worse overall survival especially if p53 is truncated [24, 25]. Nadkarni et al. showed, that TP53 null mutations presumably related to recurrent tumours. Their works indicate that deleterious alteration confers an increased and earlier probability for recurrence [26]. Furthermore, tumours with nonsense (Group B) mutations are more likely to develop metastatic tumours compared to those cancers that contain either missense mutations (Group A \& C) or are wild-type p53 [27]. Moreover, patients harbouring null TP53 alterations have an increased risk to have more vascular tumours [26]. The effect and importance of TP53 mutations are summarized in a review paper by Muller and Vousden [28]. As anticipated, more than half of the alterations $(56.5 \%)$ in the non-coding regions were mostly negative by IHC. We demonstrated that TP53 mutations with negative IHC results were most frequently caused by deletions or insertions (Table 1). Drugs inducing the read-through of early stop codons caused by mutations could be a promising therapeutic strategy of the cancer linked with non-sense mutations in tumour suppressor genes. Aminoglycoside antibiotics such as gentamicin and G418 can promote premature termination codon readthrough results in the partial restoration of full-length protein [29]. Floquet et al. described that Q192*, R213* and E298* TP53 mutations displaying high induced read-through level [30]. The aminoglycoside treatment strongly and specifically stabilized mutant p53 mRNAs that would otherwise be degraded by non-sense mediated mRNA decay [30]. Although induction of read-through of premature stop codons is effective, the clinical use of these agents is still limited by their toxicity [31].

Currently, there is no consensus as to which antibodies are most appropriate for evaluating mutationassociated p53 expression [24]. None of the routinely used p53 antibodies (CM1, Pab1801, DO1 and DO7) differentiate between mutant and wild-type $\mathrm{p} 53$ proteins $[2,21]$. While, CM1 antibody binds to the full length protein, DO7, DO1 and Pab1801 recognize epitopes only in the $\mathrm{N}$-terminus of the human $\mathrm{p} 53$ protein (amino acid residues $1-45,11-25$ and $32-79$, respectively) [3234] (Supplemental Figure S2). Importantly, the most commonly used DO7 antibody can detect only truncation mutations in exons 9-10 [24]. A further limitation of p53 IHC is that not only TP53 mutation but also disturbed p53 pathway can result in abnormal p53 expression [35]. Therefore, amplification of MDM2 or MDM4 as well as repressed $\mathrm{p} 14^{\mathrm{ARF}}$ or TP53 by promoter methylation can cause reduced p53 expression resulting in a limited sensitivity of IHC [36, 37].

Intensive efforts have been made to improve the reliability of p53 IHC as a surrogate method. Combined usage of antibodies that target various p53 epitopes or p53-related proteins, as well as quantitative scoring methods seem to be valuable approaches for TP53 mutation prediction. In 2005, Nenutil et al. applied a panel of eight antibodies that relate to p53 stabilization and transcriptional activation: anti-p53 (DO1; Bp53-10 \& Pab1801; Ser15; Ser392), anti-Ki67 (MIB1), anti-MDM2 (2A9) and anti-p21 (118) [38]. They conclude that $i$ ) overexpressed p53 without increased MDM2 indicates inactivating mutations that stabilize $\mathrm{p} 53$; ii) tumours with overexpressed $\mathrm{p} 53$ and concurrent increase of MDM2 do not have p53 mutation. iii) phosphorylated p53 expression correlates with total p53 levels and iv) does not predict TP53 mutation status [38]. Nevertheless there are no subsequent studies confirming these findings. Wertz et al. described, that a cocktail of DO1 and DO7 antibodies could identify $93 \%$ of cell lines and patient samples with TP53 missense mutations in the exons 5 to 8 region in prostatic adenocarcinoma [39]. Combined IHC of PLK1 (Polo-like kinase-1), p21, and p53 is slightly more sensitive for predicting TP53 status and may facilitate differentiation of missense and nonsense mutations [40]. The $\mathrm{p} 21$ is a transcriptional target of $\mathrm{p} 53$, therefore its expression is used for decreasing false positivity of p53 IHC. Immunohistochemical positivity of PLK1 along with negative p53 IHC can reflect nonsense TP53 mutations and may decrease the possibility of false negative IHC, because mutant p53 fails to repress PLK1 expression [41]. Köbel et al. used p53 antibodies DO7, DO1, and E26 and tagged-amplicon next generation sequencing of TP53 in high-grade serous ovarian carcinomas and endometrioid carcinomas. They demonstrated that optimized p53 IHC assay is a useful surrogate for the TP53 mutation status, and that combination of p53 IHC and sequencing should be the gold standard in assessing the p53 functional status for clinical trial inclusion [42]. IHC scoring may correlate with TP53 mutations and could increase the accuracy of p53 IHC. In a recent study, Cole et al. (2016) combined massively parallel sequencing and IHC to characterise TP53 mutations and p53 expression in highgrade serous ovarian cancer. According to their results, missense TP53 mutations have high expression of p53, whereas low expression was associated with non-missense mutations (i.e. frameshift, in-frame, nonsense, and splice). Furthermore, wild-type TP53 tumours displayed intermediate p53 IHC expression [43].

A limitation of our analysis is that we cannot predict the overall, and tumour specific sensitivity of p53 
Table 2: Comparison of p53 immunohistochemistry data with sex distribution and mean age of patients with TP53 somatic mutations based on the IARC TP53 Database (R17).

\begin{tabular}{|l|l|l|l|l|}
\hline & p53 IHC & Male & Female & $p$-value \\
\hline \multirow{3}{*}{$\begin{array}{l}\text { Mean age at } \\
\text { diagnosis (SD) }\end{array}$} & negative & $\begin{array}{l}58.567 \\
( \pm 15.062)\end{array}$ & $\begin{array}{l}55.896 \\
( \pm 15.391)\end{array}$ & $0.0182 \mathrm{a}$ \\
\cline { 2 - 5 } & positive & $\begin{array}{l}57.220 \\
( \pm 15.772)\end{array}$ & $\begin{array}{l}56.439 \\
( \pm 16.860)\end{array}$ & $0.2646 \mathrm{a}$ \\
\hline \multirow{3}{*}{ p53 IHC (\%) } & negative & $\begin{array}{l}496 \\
(23.65 \%)\end{array}$ & $\begin{array}{l}691 \\
(24.71 \%)\end{array}$ & \multirow{2}{*}{$0.8851 \mathrm{~b}$} \\
\cline { 2 - 5 } & positive & $\begin{array}{l}1601 \\
(76.35 \%)\end{array}$ & $\begin{array}{l}2106 \\
(75.29 \%)\end{array}$ & \\
\hline
\end{tabular}

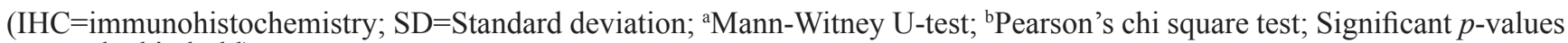
are marked in bold)

immunohistochemistry because the IARC TP53 Database does not contain IHC results of wild-type p53 expression. Consequently, the analysis and exclusion of false positive cases has not been possible. IHC antibodies are not listed in the database, therefore their specificity and association with somatic mutations could not be analysed.

In summary, the majority of TP53 mutations were missense and IHC positive, whereas most nonsense and frameshift mutations and deletions were immunonegative. Significant correlations were observed between the age at diagnosis and the immunohistochemical patterns of TP53 mutations in breast, head \& neck, bladder, liver and haematopoietic cancers. In certain tumour sites there is an increased likelihood of false negative IHC associated with rare nonsense mutations. Our frequency- and immunopositivity-based classification is useful in patient stratification and has prognostic implication.

\section{MATERIALS AND METHODS}

\section{The IARC TP53 somatic mutation database}

The last realised (R17) dataset of somatic TP53 mutation (http://p53.iarc.fr/TP53SomaticMutations. aspx) was used for the characterisation of TP53 somatic mutations, and correlate them with IHC expression data. Of the 29711 mutations, the protein description is available in 25361 mutations which are composed of 1778 unique TP53 mutations. The nomenclature is at the protein level according to the Human Genome Variation Society (HGVS) standards, using the P04637 Uniprot reference sequence.

\section{Immunohistochemical characteristics of somatic TP53 mutation}

Somatic TP53 mutations with known IHC data were retrieved and the expression patterns were analysed in various aspects, such as the localization within the TP53 gene (exons/introns, $\mathrm{CpG}$ and splice sites); effects (frameshift, intronic, large deletion, missense, nonsense, silent, splice, other); type (single nucleotide mutations, tandem, complex, deletion, insertion); and affected structural motifs of $\mathrm{p} 53$ protein (C-terminal, C-terminal/ NLS, C-terminal/tetramerization, L1/S/H2, L2/L3 loops, $\mathrm{N}$-terminal, N-terminal/Transactivation, N-terminal/ Transactivation/ NES, NDBL/ $\beta$-sheets, SH3-like/Prolinerich).

In the IARC database, p53 immunostaining is graded as 'positive' $(n=6026)$, 'negative' $(n=1852)$ and '+/-' $(n=205)$. To improve the accuracy of our study only 'positive' and 'negative' p53 IHC results were considered. Based on these criteria, of the 29711 mutations IHC data are available for 7878 TP53 somatic mutations corresponding to 1139 distinct alterations. TP53 somatic mutations frequencies and their IHC data are summarized in Supplemental Table S1. The majority of the alterations $(91.9 \%)$ are clustered within exons 5-8. More than twothirds of the mutations (68.2\%) occur in non-CpG sites, and $96.9 \%$ of the alterations are non-splice site mutations. Considering the mutation types, $6860(87.1 \%)$ are single nucleotide substitutions (SNSs), 667 (8.5\%) deletion, $196(2.5 \%)$ insertion, 97 (1.2\%) tandem and $48(0.6 \%)$ complex mutation. Mutation type was non-available only in 10 mutations $(0.1 \%)$. The most common mutation effect is missense (74.8\%), followed by frameshift $(8.6 \%)$, nonsense $(7 \%)$, silent $(4.7 \%)$, splicing $(1.7 \%)$, intronic mutations $(0.7 \%)$ and large deletion $(0.03 \%)$. Further $2.3 \%$ of the mutations is specified as 'other' and $0.4 \%$ of the alterations remains undetermined (Table 1).

\section{Clinicopathological characteristics of TP53 mutations with known IHC data}

The subset of mutations with p53 IHC results compiles data on 7124 individuals. Sex is available for 4894 individuals (62.1\%) including 2797 females (57.2\%) and 2097 males (42.9\%). The mean age at diagnosis is $56.90 \pm 16.23$ years given for 3072 patients $(62.8 \%)$ (Table 2). The available $\mathrm{p} 53 \mathrm{IHC}$ expression data are distributed among 60 tumour sites (Supplemental Table S2). Information about the sample source is available for 6957 
cases: surgery $(83.3 \%)$, biopsy $(13.5 \%)$, cell-line $(2.7 \%)$, blood $(0.3 \%)$, bone marrow $(0.1 \%)$, xenograft $(0.1 \%)$, pleural fluids $(0.03 \%)$ and saliva $(0.01 \%)$. Overall, 5211 mutations have a known origin: 4977 (95.5\%) primary tumours, $106(2 \%)$ metastasis, 84 (1.6\%) recurrent, and $44(0.8 \%)$ secondary tumours.

\section{Classification of TP53 mutations based on frequency and IHC patterns}

We investigated the frequency of 1139 distinct TP53 mutations in the IARC Database and IHC positivity of individual mutations was also calculated by dividing the IHC positive cases by the total number of the given mutation (Supplemental Table S1). Based on these characteristics, we divided TP53 somatic mutations into three groups: 1.) Group A (strongly IHC positive, hotspot mutations; mutation frequency $\geq 0.013$ and $\mathrm{IHC}$ positivity $\geq 0.85$ ), 2.) Group B (mainly IHC negative nonsense mutations; mutation frequency $\leq 0.011$ and $\mathrm{IHC}$ positivity $\leq 0.44$ ) and 3.) Group C (IHC positive, nonfrequent missense mutations; mutation frequency $\leq 0.072$ and IHC positivity $\geq 0.74$ ). Only individual mutations with 15 or more IHC results were involved in the classification. To evaluate these two components we applied principal component analysis (PCA).

\section{Statistical analysis}

Dataset of TP53 somatic mutations was downloaded and extracted using Microsoft Excel version 2013 (Microsoft Corp, Redmond, Washington, USA) and the statistical analysis was performed using SPSS 19.0 (SPSS Inc., Chicago IL) for Windows and R statistical software (www.R-project.org). Comparisons between groups were performed with Pearson's Chi-square $(\chi 2)$ test for categorical variables. Age of onset associations between groups were done with the Mann-Whitney U test. Twosided tests were computed and statistical significance was set at $p<0.05$. The difference in proportions was compared using 2-sample test for equality of proportions with continuity correction.

\section{CONFLICTS OF INTEREST}

The Authors do not have any conflicts of interest.

\section{GRANT SUPPORT}

Supported by the Hungarian Brain Research Program (NAP) - Grants No. KTIA_13_NAP-A-II/7 and KTIA_13_NAP-A-V/3, and AGR_PIAC_13-1-20130008 .

\section{REFERENCES}

1. Petitjean A, Achatz M, Borresen-Dale A, Hainaut P, Olivier M. TP53 mutations in human cancers: functional selection and impact on cancer prognosis and outcomes. Oncogene. 2007; 26: 2157-65.

2. Tan E, Cheok C. Bringing p53 into the Clinic. J Cancer Sci Ther. 2014; 6: 363-9.

3. Leroy B, Fournier JL, Ishioka C, Monti P, Inga A, Fronza G, Soussi T. The TP53 website: an integrative resource centre for the TP53 mutation database and TP53 mutant analysis. Nucleic Acids Res. 2013; 41: D962-9.

4. Kang HJ, Chun S-M, Kim K-R, Sohn I, Sung CO. Clinical relevance of gain-of-function mutations of p53 in highgrade serous ovarian carcinoma. PloS One. 2013; 8: e72609.

5. Schlomm T, Iwers L, Kirstein P, Jessen B, Köllermann J, Minner S, Passow-Drolet A, Mirlacher M, Milde-Langosch $\mathrm{K}$, Graefen M. Clinical significance of p53 alterations in surgically treated prostate cancers. Mod Pathol. 2008; 21: 1371-8.

6. Dobes P, Podhorec J, Coufal O, Jureckova A, Petrakova K, Vojtesek B, Hrstka R. Influence of mutation type on prognostic and predictive values of TP53 status in primary breast cancer patients. Oncol Rep. 2014; 32: 1695-702.

7. Robles AI, Harris CC. Clinical outcomes and correlates of TP53 mutations and cancer. Cold Spring Harb Perspect Biol. 2010; 2: a001016.

8. Boyd SD. Diagnostic applications of high-throughput DNA sequencing. Annu Rev Pathol Mech Dis. 2013; 8: 381-410.

9. Yemelyanova A, Vang R, Kshirsagar M, Lu D, Marks MA, Shih IM, Kurman RJ. Immunohistochemical staining patterns of p53 can serve as a surrogate marker for TP53 mutations in ovarian carcinoma: an immunohistochemical and nucleotide sequencing analysis. Mod Pathol. 2011; 24: 1248-53.

10. Ando K, Oki E, Saeki H, Yan Z, Tsuda Y, Hidaka G, Kasagi Y, Otsu H, Kawano H, Kitao H. Discrimination of p53 immunohistochemistry-positive tumors by its staining pattern in gastric cancer. Cancer Med. 2015; 4: 75-83.

11. Temam S, Flahault A, Périé S, Monceaux G, Coulet F, Callard P, Bernaudin J-F, St Guily JL, Fouret P. p53 gene status as a predictor of tumor response to induction chemotherapy of patients with locoregionally advanced squamous cell carcinomas of the head and neck. J Clin Oncol. 2000; 18: 385-385.

12. Salnikova LE. Clinicopathologic Characteristics of Brain Tumors are Associated with the Presence and Patterns of TP53 Mutations: Evidence from the IARC TP53 Database. Neuromolecular Med. 2014; 16: 431-47.

13. Edlund K, Larsson O, Ameur A, Bunikis I, Gyllensten U, Leroy B, Sundström M, Micke P, Botling J, Soussi T. Datadriven unbiased curation of the TP53 tumor suppressor gene mutation database and validation by ultradeep sequencing 
of human tumors. Proc Natl Acad Sci. 2012; 109: 9551-6.

14. Bouaoun L, Sonkin D, Ardin M, Hollstein M, Byrnes G, Zavadil J, Olivier M. TP53 Variations in Human Cancers: New Lessons from the IARC TP53 Database and Genomics Data. Hum Mutat. 2016; 37(9): 865-76.

15. López I, Oliveira LP, Tucci P, Álvarez-Valín F, Coudry RA, Marín M. Different mutation profiles associated to P53 accumulation in colorectal cancer. Gene. 2012; 499: 81-7.

16. Bouchalova P, Nenutil R, Muller P, Hrstka R, Appleyard M, Murray K, Jordan LB, Purdie CA, Quinlan P, Thompson AM. Mutant p53 accumulation in human breast cancer is not an intrinsic property or dependent on structural or functional disruption but is regulated by exogenous stress and receptor status. J Pathol. 2014; 233: 238-46.

17. Silwal-Pandit L, Vollan HKM, Chin S-F, Rueda OM, McKinney S, Osako T, Quigley DA, Kristensen VN, Aparicio S, Børresen-Dale A-L. TP53 mutation spectrum in breast cancer is subtype specific and has distinct prognostic relevance. Clin Cancer Res. 2014; 20: 3569-80.

18. Vakiani E, Janakiraman M, Shen R, Sinha R, Zeng Z, Shia J, Cercek A, Kemeny N, D’Angelica M, Viale A. Comparative genomic analysis of primary versus metastatic colorectal carcinomas. J Clin Oncol. 2012; 30: 2956-62.

19. Bromidge T, Lowe C, Prentice A, Johnson S. p53 intronic point mutation, aberrant splicing and telomeric associations in a case of B-chronic lymphocytic leukaemia. Br J Haematol. 2000; 111: 223-9.

20. Holmila R, Fouquet C, Cadranel J, Zalcman G, Soussi T. Splice mutations in the $\mathrm{p} 53$ gene: case report and review of the literature. Hum Mutat. 2003; 21: 101-2.

21. Lee SH, Kim H, Kim WY, Han HS, Lim SD, Kim WS, Kim S, Hwang TS. Genetic alteration and immunohistochemical staining patterns of ovarian highgrade serous adenocarcinoma with special emphasis on p53 immnnostaining pattern. Pathol Int. 2013; 63: 252-9.

22. Evans DGR. Neurofibromatosis 2 [Bilateral acoustic neurofibromatosis, central neurofibromatosis, NF2, neurofibromatosis type II]. Genet Med. 2009; 11: 599-610.

23. Bellanné-Chantelot C, Carette C, Riveline J-P, Valéro R, Gautier J-F, Larger E, Reznik Y, Ducluzeau P-H, Sola A, Hartemann-Heurtier A. The type and the position of HNF1A mutation modulate age at diagnosis of diabetes in patients with maturity-onset diabetes of the young (MODY)-3. Diabetes. 2008; 57: 503-8.

24. Shahin MS, Hughes JH, Sood AK, Buller RE. The prognostic significance of p53 tumor suppressor gene alterations in ovarian carcinoma. Cancer. 2000; 89: 200617.

25. Hashimoto T, Tokuchi $\mathrm{Y}$, Hayashi M, Kobayashi Y, Nishida K, Hayashi S, Ishikawa Y, Tsuchiya S, Nakagawa K, Hayashi J. p53 null mutations undetected by immunohistochemical staining predict a poor outcome with early-stage non-small cell lung carcinomas. Cancer Res.
1999; 59: 5572-7.

26. Nadkarni NJ, De Geest K, Neff T, De Young B, Bender DP, Ahmed A, Smith BJ, Button A, Goodheart MJ. Microvessel density and p53 mutations in advanced-stage epithelial ovarian cancer. Cancer Lett. 2013; 331: 99-104.

27. Sood AK, Sorosky JI, Dolan M, Anderson B, Buller RE. Distant metastases in ovarian cancer: association with p53 mutations. Clin Cancer Res. 1999; 5: 2485-90.

28. Muller PA, Vousden KH. p53 mutations in cancer. Nat Cell Biol. 2013; 15: 2-8.

29. Zomer-van Ommen D, Vijftigschild L, Kruisselbrink E, Vonk A, Dekkers J, Janssens H, de Winter-de Groot K, van der Ent C, Beekman J. Limited premature termination codon suppression by read-through agents in cystic fibrosis intestinal organoids. J Cyst Fibros. 2016; 15: 158-62.

30. Floquet C, Deforges J, Rousset J-P, Bidou L. Rescue of non-sense mutated p53 tumor suppressor gene by aminoglycosides. Nucleic Acids Res. 2011; 39: 3350-62.

31. Soussi T, Wiman K. TP53: an oncogene in disguise. Cell Death Differ. 2015; 22: 1239-49.

32. Danks MK, Whipple DO, McPake CR, Lu D, Harris LC. Differences in epitope accessibility of p53 monoclonal antibodies suggest at least three conformations or states of protein binding of p53 protein in human tumor cell lines. Cell Death Differ. 1998; 5.

33. Phang BH, Othman R, Bougeard G, Chia RH, Frebourg T, Tang CL, Cheah PY, Sabapathy K. Amino-terminal p53 mutations lead to expression of apoptosis proficient p47 and prognosticate better survival, but predispose to tumorigenesis. Proc Natl Acad Sci. 2015; 112: E6349-58.

34. Christgen M, Noskowicz M, Heil C, Schipper E, Christgen H, Geffers R, Kreipe H, Lehmann U. IPH-926 lobular breast cancer cells harbor a p53 mutant with temperaturesensitive functional activity and allow for profiling of p53responsive genes. Lab Invest. 2012; 92: 1635-47.

35. Tanboon J, Williams EA, Louis DN. The Diagnostic use of immunohistochemical surrogates for signature molecular genetic alterations in gliomas. J Neuropathol Exp Neurol. 2016; 75: 4-18.

36. Manfredi JJ. The Mdm2-p53 relationship evolves: Mdm2 swings both ways as an oncogene and a tumor suppressor. Genes Dev. 2010; 24: 1580-9.

37. Theodorescu D. New and promising strategies in the management of bladder cancer. American Society of Clinical Oncology; 2015.

38. Nenutil R, Smardova J, Pavlova S, Hanzelkova Z, Muller P, Fabian P, Hrstka R, Janotova P, Radina M, Lane D. Discriminating functional and non-functional p53 in human tumours by p53 and MDM2 immunohistochemistry. J Pathol. 2005; 207: 251-9.

39. Wertz IE, Deitch AD, Gumerlock PH, Gandour-Edwards R, Chi S-G, de Vere White RW. Correlation of genetic and immunodetection of TP53 mutations in malignant and 
benign prostate tissues. Hum Pathol. 1996; 27: 573-80.

40. Watanabe G, Ishida T, Furuta A, Takahashi S, Watanabe M, Nakata H, Kato S, Ishioka C, Ohuchi N. Combined Immunohistochemistry of PLK1, p21, and p53 for Predicting TP53 Status. Am J Surg Pathol. 2015; 39: 102634.

41. King SI, Purdie CA, Bray SE, Quinlan PR, Jordan LB, Thompson AM, Meek DW. Immunohistochemical detection of Polo-like kinase-1 (PLK1) in primary breast cancer is associated with TP53 mutation and poor clinical outcom. Breast Cancer Res. 2012; 14: R40.
42. Köbel M, Maria Piskorz A, Lee S, Lui S, LePage C, Marass F, Rosenfeld N, Mes Masson A, Derek Brenton J. Optimized p53 immunohistochemistry is an accurate predictor of TP53 mutation in ovarian carcinoma. J Pathol Clin Res. 2016; .

43. Cole AJ, Dwight T, Gill AJ, Dickson K-A, Zhu Y, Clarkson A, Gard GB, Maidens J, Valmadre S, Clifton-Bligh R. Assessing mutant $\mathrm{p} 53$ in primary high-grade serous ovarian cancer using immunohistochemistry and massively parallel sequencing. Sci Rep. 2016; 6. 